\title{
DIVERSITY RECEPTION OVER CORRELATED RICEAN FADING SATELLITE CHANNELS
}

Petros S. Bithas and P. Takis Mathiopoulos

Institute for Space Applications and Remote Sensing, National Observatory of Athens,

Metaxa \& Vas. Pavlou Street, 15236 Athens, Greece E-mail: \{pbithas;mathio\}@space.noa.gr

\begin{abstract}
In this paper, the performance of switch and stay combing (SSC) diversity receivers operating over correlated Ricean fading channels, is studied. By representing the bivariate Ricean distribution as infinite series the probability density function (PDF) of the SSC output signal-to-noise ratio (SNR) has been derived. Capitalizing on this PDF the moments of the output SNR and the corresponding cumulative distribution function $(C D F)$ have been also obtained. Furthermore, an analytical expression for the moments generating function $(M G F)$ is derived and by employing the MGF-based approach the average bit error probability (ABEP) for several modulation schemes is studied. Finally, various performance evaluation results, such as average output SNR (ASNR), amount of fading $(A o F)$, outage probability $\left(P_{\text {out }}\right)$ and $A B E P$ are presented.
\end{abstract}

\section{INTRODUCTION}

The mobile radio channel is particularly dynamic due to multipath propagation, which has a strong negative impact on the average bit error probability (ABEP) of any modulation technique [1]. Diversity is a powerful communication receiver technique used to compensate for fading channel impairments. The most important diversity reception methods employed in digital communication receivers are maximal-ratio combining (MRC), equal gain combining (EGC), selection combining (SC) and switch and stay combing (SSC) [2]. Among them, SSC diversity is the least complex and can be used in conjunction with coherent, non-coherent and differentially coherent modulation schemes. It is well known that in many real life communication scenarios the combined signals are correlated [2]. A typical example for such signal correlation exists in small size mobile terminal units where the distance between diversity antenna is small. Due to this correlation between the signals received at the diversity branches, there is degradation in the diversity gain. As far as the Ricean distribution is concerned, it is often used to model propagation paths consisting of one strong direct line-of-sight (LoS) signal and many random reflected and usually weaker signals. Such fading environments are typically observed in microcellular and mobile satellite radio links [2]. Especially for mobile satellite communications, the Ricean distribution is used to accurately characterize the mobile satellite channel, for the 
single-state [3] and the clear state [4]. Moreover, in [5] it was depicted that the Ricean- $K$ factor characterizes the land mobile satellite channel during unshadowed periods.

The technical literature concerning diversity receivers operating over correlated fading channels is quite extensive, e.g., [6-9]. In [6] expressions for the outage probability $\left(P_{\text {out }}\right)$ and average bit error probability (ABEP) of dual SC with correlated Rayleigh, were derived either in closed-form or in terms of single integrals. In [7] the cumulative distribution functions (CDF) of SC, in correlated Rayleigh, Ricean and Nakagami- $m$ fading channels were derived in terms of single fold integral and infinite series expressions. In [8] the ABEP of dual-branch EGC and MRC receivers was obtained in correlative Weibull fading. More recently, in [9] the performance of MRC in nonidentical Weibull fading channels with arbitrary parameters was evaluated.

Past work concerning the performance of SSC operating over correlated fading channels can be found in [10-13]. One of the first attempts to investigate the performance of SSC diversity receivers operating on independent and correlated Ricean fading channels was made in [10]. However, in this reference only a integral representation for the ABEP using non-coherent frequency shift keying (NCFSK), has been obtained. In [11] the performance of SSC diversity receivers was evaluated and optimized for several channel conditions, including different fading channels and unbalanced branches fading correlation. However, the research reported in [11] was restricted to correlated Nakagami- $m$ fading conditions. In [12] the moment generating function (MGF) of SSC is derived in finite integral representation and has been also applied to correlated Nakagami- $m$ channel. Recently in [13] analytical results for the performance of non ideal referenced based SSC for $M$-ary digitally modulated signals in correlated Nakagami- $m$ fading channels were derived. Although previous research on generalized fading channels is rather extensive, the problem of analyzing the performance of SSC over correlated Ricean fading channel has not been adequately addressed. The main difficulty for this is the complicated form of the bivariate probability density function (PDF) derived in [10] and the absence of an alternative expression for the multivariate distribution. On the contrary as it will agreed in our paper by using a PDF of the form derived in [14], the most important statistic metrics of SSC output signal to noise ratio (SNR) can be obtained.

The remainder of this paper is organized as follows. In Section 1 the system and channel model are presented. In Section 2 the statistics and the most important performance metrics of the SSC output SNR are derived while in Section 3 some numerical evaluation results are given. 


\section{SYSTEM AND CHANNEL MODEL}

Let us consider a dual-branch SSC diversity receiver operating over a correlated Ricean fading channel. The baseband receiver signal at the $\ell$ th $(\ell=1,2)$ input branch is $\zeta_{\ell}=s h_{\ell}+n_{\ell}$, where $s$ is the complex transmitted symbol, $E_{s}=E\left\langle|s|^{2}\right\rangle$ is the transmitted average symbol energy, where $E\langle\cdot\rangle$ denotes expectation and $|\cdot|$ absolute value, $h_{\ell}$ is the complex channel fading envelope, with its magnitude $R_{\ell}=\left|h_{\ell}\right|$, and $n_{\ell}$ is the additive white Gaussian noise (AWGN) with single-sided power spectral density $N_{0}$.

The instantaneous SNR per symbol at the $\ell$ th input branch is $\gamma_{\ell}=R_{\ell} E_{s} /\left(2 N_{0}\right)$ and the corresponding average SNR per symbol at both input branches is $\bar{\gamma}=\Omega E_{s} / N_{0}$, where $\Omega=E\left\langle R_{\ell}^{2}\right\rangle$. Using a similar procedure as for deriving [14, eq. (9)], the joint PDF of $\gamma_{1}$ and $\gamma_{2}$ can be obtained as

$$
\begin{aligned}
f_{\gamma_{1}, \gamma_{2}}\left(\gamma_{1}, \gamma_{2}\right)= & \sum_{\substack{i, h=0 \\
v_{1}+v_{2}+v_{3}=i}}^{\infty} \mathcal{A} \exp \left[-\beta_{1}\left(\gamma_{1}+\gamma_{2}\right)\right] \\
& \times\left(\mathcal{B} \gamma_{1}^{\beta_{2}-1} \gamma_{2}^{\beta_{3}-1}+\mathcal{C} \bar{\gamma}^{-1} \gamma_{1}^{\beta_{2}-1 / 2} \gamma_{2}^{\beta_{3}-1 / 2}\right)
\end{aligned}
$$

where

$$
\begin{gathered}
\mathcal{A}=\frac{2^{v_{3}+2 h-1}(1+K)^{1+\beta_{4}} \rho^{2 h} K^{i} \exp \left(-\frac{2 K}{1+\rho}\right)}{\sqrt{\pi} \bar{\gamma}^{1+\beta_{4}}\left(1-\rho^{2}\right)^{1+2 h} v_{1} ! v_{2} ! v_{3} ! i !(1+\rho)^{2 i}}, \\
\mathcal{B}=\frac{\left[1+(-1)^{v_{3}}\right] \Gamma\left[h+\left(1+v_{3}\right) / 2\right]}{\Gamma\left[h+1+v_{3} / 2\right] \Gamma(1+2 h)}, \\
\mathcal{C}=\frac{\left[-1+(-1)^{v_{3}}\right] 2 \rho(1+K) \Gamma\left(1+h+v_{3} / 2\right)}{\left(\rho^{2}-1\right) \Gamma(2+2 h) \Gamma\left[h+\left(3+v_{3}\right) / 2\right]}, \\
\beta_{1}=\frac{(1+K)}{\left(1-\rho^{2}\right) \bar{\gamma}}, \beta_{2}=v_{1}+\frac{v_{3}}{2}+h+1, \\
\beta_{3}=v_{2}+\frac{v_{3}}{2}+h+1, \beta_{4}=i+2 h+1
\end{gathered}
$$

where $\Gamma(\cdot)$ is the Gamma function [15, eq. (8.310/1)], $K$ is the Ricean factor, defined as the ratio of the specular signal power to the scattered power, $\rho$ is the correlation coefficient between $\gamma_{1}$ and $\gamma_{2}$. In [14] was proved that the infinity series expression in (2) converge always and converge rapidly.

\section{STATISTICS AND PERFORMANCE ANALYSIS}

In this section the most important statistical metrics of SSC diversity receivers and a detailed performance analysis for these receivers operating over correlated Ricean fading channels will be presented. 


\subsection{Probability Density Function (PDF)}

Let $\gamma_{s s c}$ be the instantaneous SNR per symbol at the output of the SSC and $\gamma_{\tau}$ the predetermined switching threshold. By using [11], the PDF of $\gamma_{s s c}$, $f_{\gamma_{s s c}}(x)$, can be obtained as follows

$$
f_{\gamma_{s s c}}(x)= \begin{cases}r_{s s c}(x), & x \leq \gamma_{\tau} \\ r_{s s c}(x)+f_{\text {Rice }}(x), & x>\gamma_{\tau}\end{cases}
$$

where

$$
f_{\text {Rice }}(x)=\frac{1+K}{\bar{\gamma}} \exp (-K) \exp \left[-\frac{(1+K)}{\bar{\gamma}} x\right] I_{0}\left(2 \sqrt{\frac{K(K+1)}{\bar{\gamma}}} x^{1 / 2}\right)
$$

with $I_{0}(\cdot)$ being the zeroth-order modified Bessel function of the first kind [15, eq. (8.406)]. Moreover, using [11, eq. (70)], [15, eq. (3.351)], $r_{s s c}(x)$ can be obtained as

$$
\begin{aligned}
r_{s s c}(x)= & \sum_{\substack{i, h=0 \\
v_{1}+v_{2}+v_{3}=i}}^{\infty} \mathcal{A} \exp \left(-\beta_{1} x\right) x^{\beta_{2}-1 / 2} \\
& \times\left[\frac{\mathcal{B}}{\sqrt{x} \beta_{1}^{\beta_{3}}} \gamma\left(\beta_{3}, \beta_{1} \gamma_{\tau}\right)+\frac{\mathcal{C}}{\bar{\gamma} \beta_{1}^{\beta_{3}+1 / 2}} \gamma\left(\beta_{3}+1 / 2, \beta_{1} \gamma_{\tau}\right)\right]
\end{aligned}
$$

where $\gamma(\cdot, \cdot)$ is the lower incomplete Gamma function [15, eq. (8.350)].

\subsection{Cumulative Density Function (CDF) and Outage Probability $\left(P_{\text {out }}\right)$}

Using [16, eq. (20)], the CDF of $\gamma_{s s c}$ can be obtained as

$$
\begin{aligned}
F_{\gamma_{s s c}}(x)= & \operatorname{Pr}\left(\gamma_{\tau} \leq \gamma_{1} \leq x\right) \\
& +\operatorname{Pr}\left(\gamma_{2}<\gamma_{\tau} \text { and } \gamma_{1}<x\right) .
\end{aligned}
$$

After some manipulations, (4) can be expressed in terms of CDF's as

$$
F_{\gamma_{s s c}}(x)= \begin{cases}F_{\gamma_{1}, \gamma_{2}}\left(x, \gamma_{\tau}\right), & x \leq \gamma_{\tau} \\ F_{\gamma}(x)-F_{\gamma}\left(\gamma_{\tau}\right)+F_{\gamma_{1}, \gamma_{2}}\left(x, \gamma_{\tau}\right), & x>\gamma_{\tau}\end{cases}
$$

with

$$
F_{\gamma}(x)=Q_{1}\left[\sqrt{2 K}, \sqrt{\frac{2(1+K)}{\bar{\gamma}} x}\right]
$$

where $Q_{1}(\cdot)$ is the first order Marcum-Q function [2, eq. (4.33)]. In (5) $F_{\gamma_{1}, \gamma_{2}}\left(x, \gamma_{\tau}\right)$ can be derived by using [15, eq. (3.352)] as

$$
\begin{aligned}
F_{\gamma_{1}, \gamma_{2}}\left(x, \gamma_{\tau}\right)= & \frac{\mathcal{A}}{\beta_{1}^{\beta_{2}+\beta_{3}}}\left[\mathcal{B} \gamma\left(\beta_{2}, \beta_{1} x\right) \gamma\left(\beta_{3}, \beta_{1} \gamma_{\tau}\right)\right. \\
& \left.+\frac{\mathcal{C}}{\beta_{1}} \gamma\left(\beta_{2}+1 / 2, \beta_{1} x\right) \gamma\left(\beta_{3}+1 / 2, \beta_{1} \gamma_{\tau}\right)\right] .
\end{aligned}
$$


Clearly, the probability that the output SNR falls below a given threshold $\left(\gamma_{t h}\right), P_{\text {out }}$, can be obtained for $\gamma_{s s c}$ as

$$
P_{\text {out }}\left(\gamma_{t h}\right)=F_{\gamma_{s s c}}\left(\gamma_{t h}\right) \text {. }
$$

\subsection{Moments Generating Function (MGF) and Average Bit Error Probability (ABEP)}

Based on (2) the MGF for $\gamma_{s s c}, \mathcal{M}_{\gamma_{s s c}}(s)$, defined in [17, eq. (5.62)], can be expressed in terms of two integrals as

$$
\begin{aligned}
\mathcal{M}_{\gamma_{s s c}}(s) & =\int_{0}^{\infty} \exp (-s x) r_{s s c}(x) d x+\int_{\gamma_{\tau}}^{\infty} \exp (-s x) f_{\text {Rice }}(x) d x \\
& =\mathcal{I}_{1}+\mathcal{I}_{2} .
\end{aligned}
$$

Using [15, eq. (3.381/4)], $\mathcal{I}_{1}$ can be solved as

$$
\begin{aligned}
\mathcal{I}_{1}=\mathcal{A}[ & \frac{\Gamma\left(\beta_{2}\right)}{\left(\beta_{1}+s\right)^{\beta_{2}}} \mathcal{B} \beta_{1}^{\beta_{3}} \gamma\left(\beta_{3}, \beta_{1} \gamma_{\tau}\right) \\
& \left.+\mathcal{C} \beta_{1}^{\beta_{3}-1 / 2} \frac{\Gamma\left(\beta_{2}+1 / 2\right)}{\left(\beta_{1}+s\right)^{\beta_{2}+1 / 2}} \gamma\left(\beta_{3}+1 / 2, \beta_{1} \gamma_{\tau}\right)\right] .
\end{aligned}
$$

Setting $\psi=\sqrt{2 x\left(\frac{1+K}{\bar{\gamma}}+s\right)}$ and using again [2, eq. (4.33)], $\mathcal{I}_{2}$ can be solved as

$$
\begin{aligned}
\mathcal{I}_{2}=Q_{1} & \left(\sqrt{\frac{2 K(1+K)}{1+K+\bar{\gamma} s}}, \sqrt{\frac{2(1+K+\bar{\gamma} s) \gamma_{\tau}}{\bar{\gamma}}}\right) \\
& \times \exp \left(\frac{K(1+K)}{1+K+\bar{\gamma} s}\right) \frac{(1+K) \exp (-K)}{1+K+\bar{\gamma} s} .
\end{aligned}
$$

Using (8), (9) and (10) and based on the well-known MGF approach [2, 18], the ABEP for several coherent and non-coherent modulation schemes can be obtained.

\subsection{Moments, Average Output SNR (ASNR) and Amount of Fading (AoF)}

Based on (2) the moments for $\gamma_{s s c}, \mu_{\gamma_{s s c}}(n)$, defined in [17, eq. (5.38)], can be expressed in terms of two integrals as

$$
\begin{aligned}
\mu_{\gamma_{s s c}}(n) & =\int_{0}^{\infty} x^{n} r_{s s c}(x) d x+\int_{\gamma_{\tau}}^{\infty} x^{n} f_{\text {Rice }}(x) d x \\
& =\mathcal{I}_{3}+\mathcal{I}_{4} .
\end{aligned}
$$




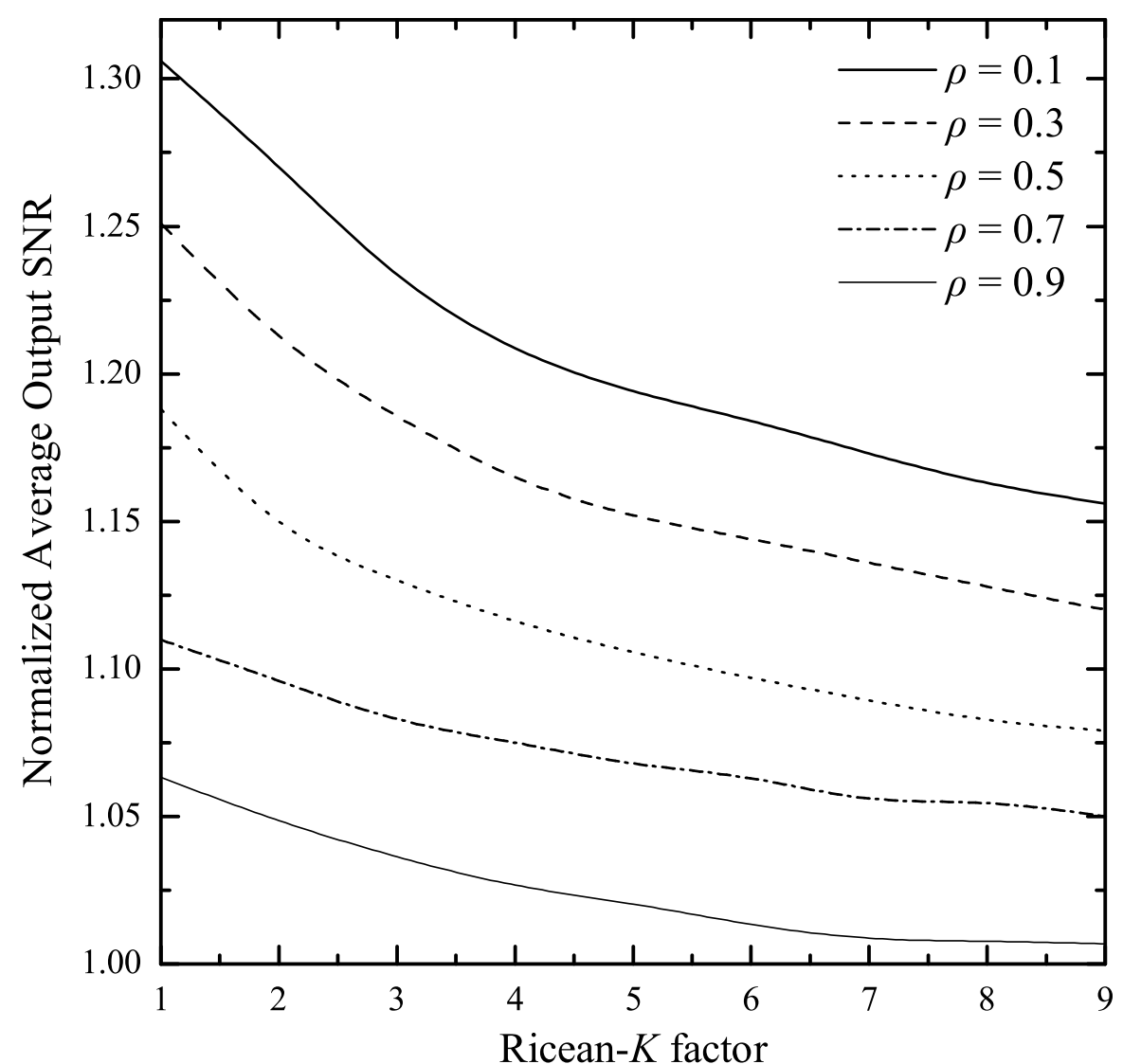

Figure 1. Normalized average output SNR (ASNR) versus the Ricean- $K$ factor for several values of $\rho$.

Using [15, eq. (3.381/4)], $\mathcal{I}_{3}$ can be easily solved as

$$
\begin{aligned}
\mathcal{I}_{3}=\mathcal{A}\left[\mathcal{B} \beta_{1}^{-\beta_{3}} \gamma\left(\beta_{3}, \beta_{1} \gamma_{\tau}\right) \frac{\Gamma\left(n+\beta_{2}\right)}{\beta_{1}^{n+\beta_{2}}}\right. \\
\left.+\frac{\mathcal{C} \gamma\left(\beta_{3}+1 / 2, \beta_{1} \gamma_{\tau}\right)}{\beta_{1}^{\beta_{3}+1 / 2}} \frac{\Gamma\left(n+\beta_{2}+1 / 2\right)}{\beta_{1}^{n+\beta_{2}+1 / 2}}\right] .
\end{aligned}
$$

Setting $\phi=\sqrt{2 \frac{1+K}{\bar{\gamma}} x}$ in $\mathcal{I}_{4}$ and after some straight forward mathematical manipulations yields

$$
\mathcal{I}_{4}=\frac{\bar{\gamma}^{n} \exp (K)}{2^{n}(1+K)^{n}} Q_{2 n+1,0}\left(K, \sqrt{\frac{2(1+K) \gamma_{\tau}}{\bar{\gamma}}}\right)
$$

where $Q_{m, n}(\cdot, \cdot)$ is the Nuttal Q-function defined in [2, eq. (4.104)]. The ASNR, $\bar{\gamma}_{\text {out }}$, is a useful performance measure serving as an excellent indi- 


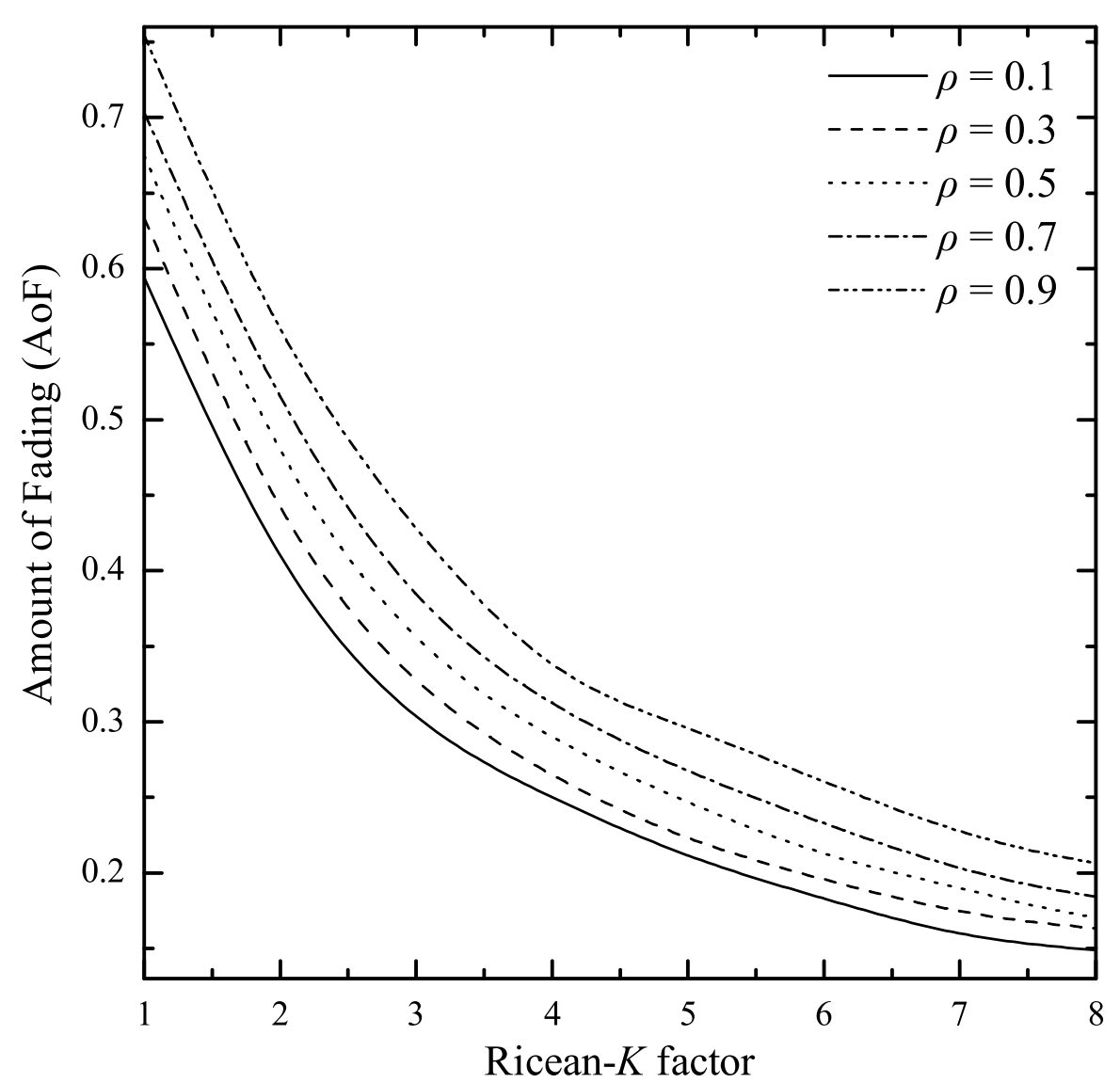

Figure 2. Amount of Fading (AoF) versus the Ricean- $K$ factor for several values of $\rho$.

cator for the overall system fidelity. The AoF, defined as $\mathrm{AoF} \triangleq \operatorname{var}(\mathrm{x}) / \bar{\gamma}^{2}$, is a unified measure of the severity of the fading channel [2], which can be expressed in terms of first- and second-order moments of $\gamma_{s s c}$ as

$$
\mathrm{AoF}=\frac{\mu_{\gamma_{\mathrm{ssc}}}(2)}{\mu_{\gamma_{\mathrm{ssc}}}(1)^{2}}-1 .
$$

Both these important performance metrics can be derived by using (11), (12) and (13).

\section{NUMERICAL PERFORMANCE EVALUATION RESULTS}

In this section, by using the previous mathematical analysis, performance evaluation results obtained by means of numerical techniques are presented. These results include different correlated Ricean fading conditions and several modulation formats. 


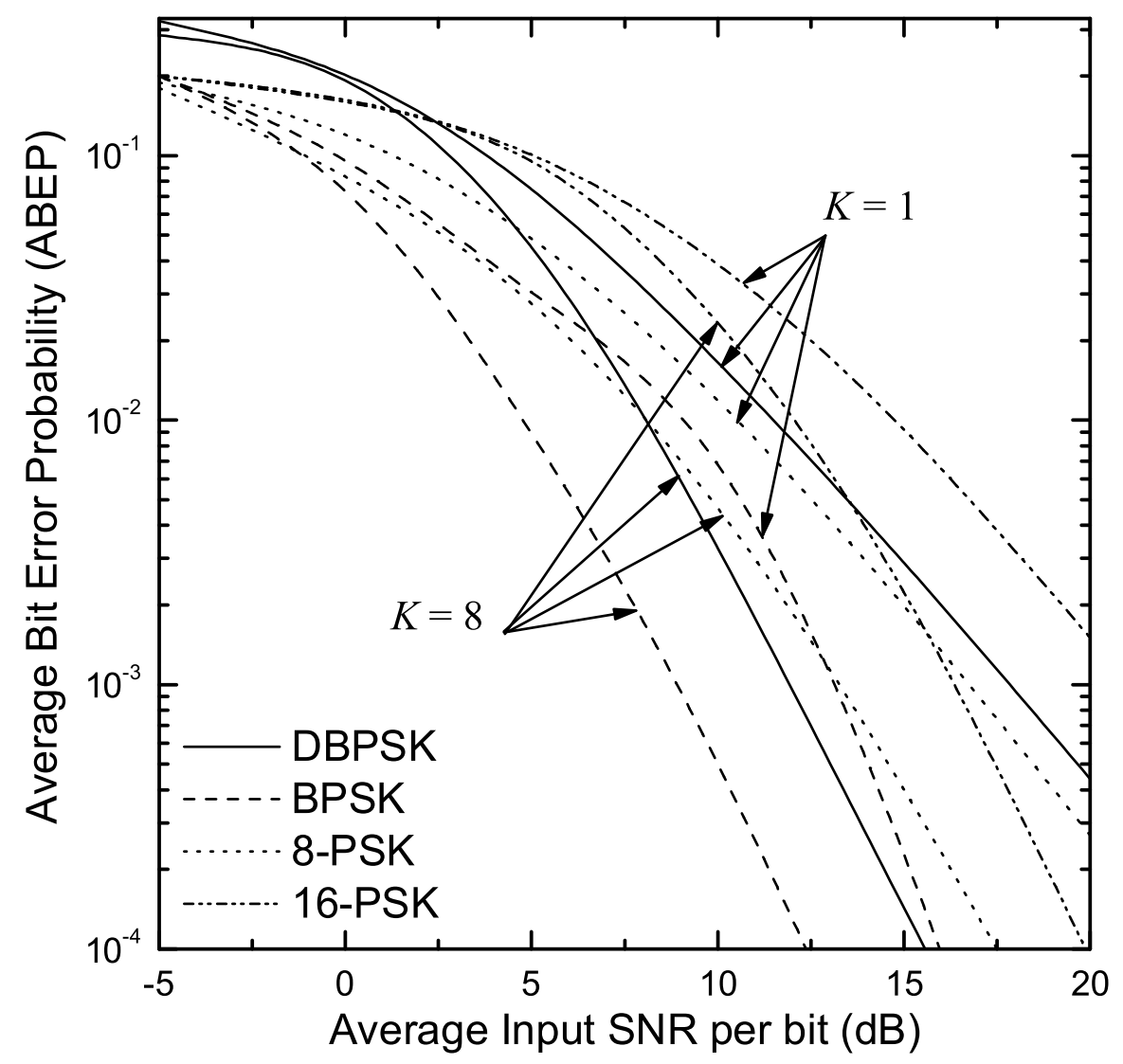

Figure 3. Average bit error probability (ABEP) versus the average input SNR per bit for DBPSK and $M$-PSK $(M=8,16)$ signaling formats for different values of the Ricean- $K$ factor.

In Figs. 1 and 2 the ASNR and AoF are plotted as a function of the Ricean- $K$ factor for several values of the correlation coefficient $\rho$. In Fig. 1 as $K$ and/or $\rho$ increase the ASNR decreases, which means that the diversity gain lessens. In Fig. 2 as $K$ increases and/or $\rho$ decreases the AoF lessens.

In Figs. 3 and 4 the ABEP is plotted as a function of the average input SNR per bit, i.e., $\bar{\gamma}_{b}=\bar{\gamma} / \log _{2} M$, for several values of $K$. In Fig. 3 the differential binary phase shift keying (DBPSK), binary phase shift keying (BPSK) and $M$-ary phase shift keying ( $M$-PSK) (gray encoding signals are considered) signaling formats are employed. It is depicted that as $K$ increases the ABEP improves and the BPSK has the best performance. In Fig. 4 considering the 16- quadrature amplitude modulation (QAM) scheme, it can been obtained as $K$ increases and/or $\rho$ decreases the ABEP lessens. 


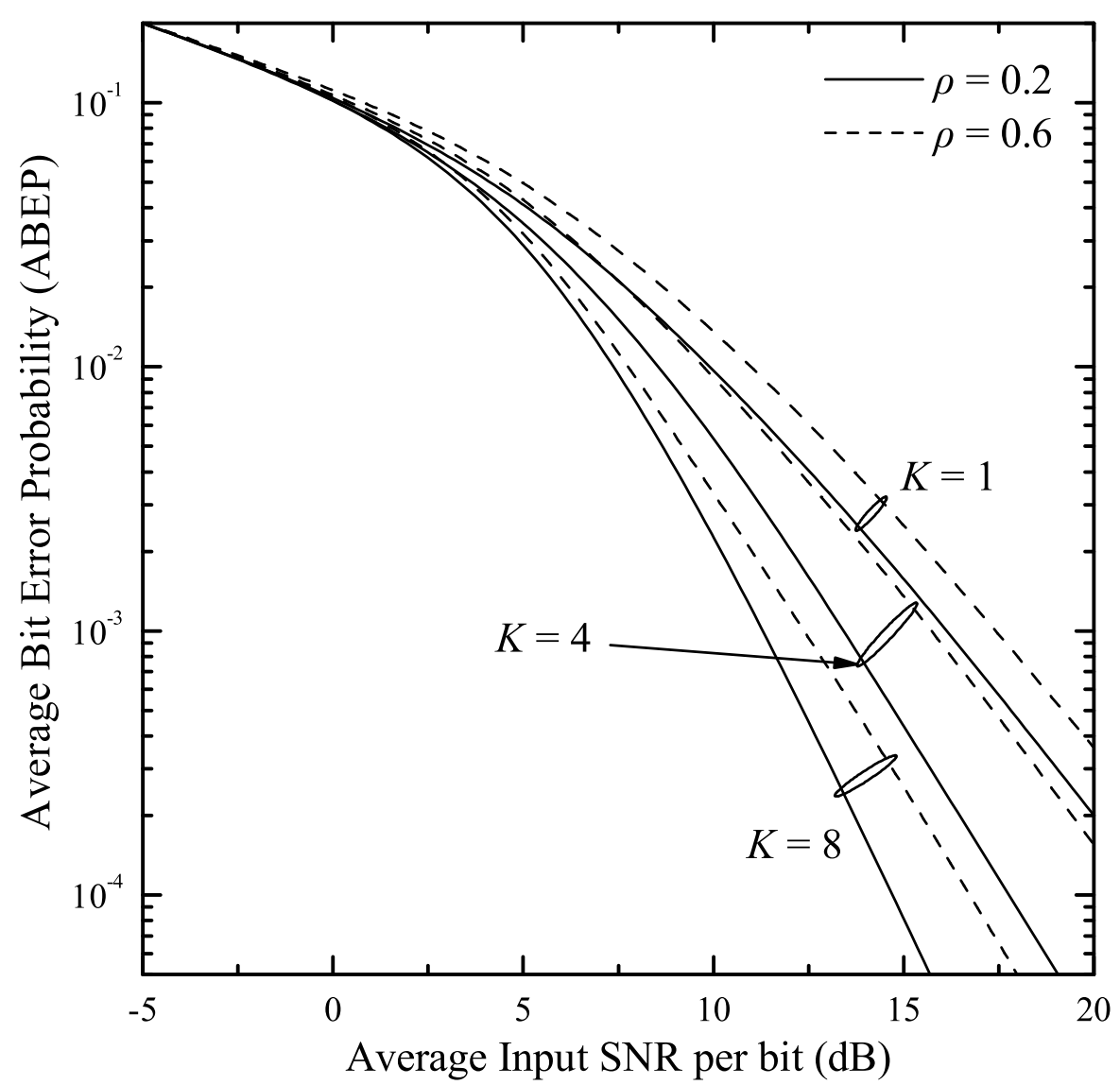

Figure 4. Average bit error probability (ABEP) versus the average input SNR per bit for 16 -QAM signaling format for different values of the Ricean- $K$ factor and $\rho$.

Finally, in Fig. 5 the $P_{\text {out }}$ is plotted as a function of the normalized outage threshold $\gamma_{t h} / \bar{\gamma}_{b}$ for several values of $K$ and $\rho$. As $K$ increases and/or $\rho$ decreases the $P_{\text {out }}$ lessens.

\section{ACKNOWLEDGMENTS}

This work has been performed within the framework of the Satellite Network of Excellence (SatNEx-II) project (IST-027393), a Network of Excellence (NoE) funded by European Commission (EC) under the FP6 program.

\section{References}

[1] T. S. Rappaport, Wireless Communications. New Jersey: Prentice Hall PTR, 2002.

[2] M. K. Simon and M.-S. Alouini, Digital Communication over Fading Channels, 2nd ed. New York: Wiley, 2005. 


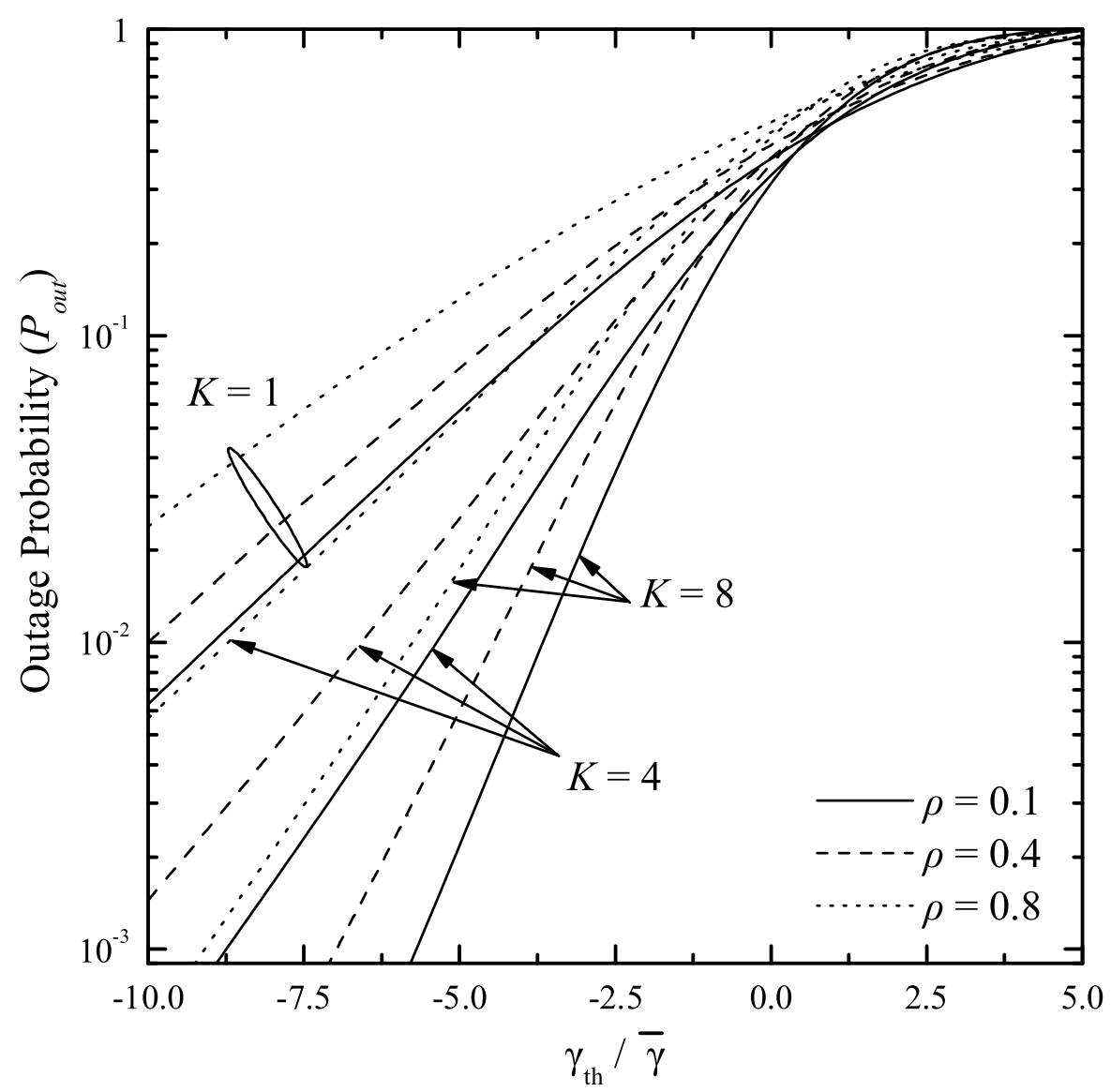

Figure 5. Outage probability versus the normalized average input SNR for several values of the Ricean- $K$ factor and $\rho$.

[3] G. E. Corazza and F. Vatalaro, "A statistical model for land mobile satellite channels and its applications to nongeostationary orbit system," IEEE Trans. Veh. Technol., vol. 43, no. 3, pp. 738-742, Aug. 1994.

[4] H. Wakana, "A propagation model for land-mobile-satellite communication," in Proc. IEEE Antennas and Propagation Sociaty International Symposium, vol. 3, June 1991, pp. $1526-1529$.

[5] E. Lutz, D. Cygan, M. Dippold, F. Dolainsky, and W. Papke, "The land mobile satellite communication channel-recording, statistics, and channel model," IEEE Trans. Veh. Technol., vol. 40, no. 2, pp. 375-386, May 1991.

[6] M. K. Simon and M.-S. Alouini, "A unified performance analysis of digital communications with dual selective combining diversity over correlated Rayleigh and Nakagami-m fading channels," IEEE Trans. Commun., vol. 47, no. 1, pp. 33-43, Jan. 1999.

[7] Y. Chen and C. Tellambura, "Distribution functions of selection combiner output in equally correlated Rayleigh, Rician, and Nakagami-m fading channels," IEEE Trans. Commun., vol. 52, no. 11, pp. 1948-1956, Nov. 2004.

[8] G. K. Karagiannidis, D. A. Zogas, N. C. Sagias, S. A. Kotsopoulos, and G. S. Tombras, "Equal-gain and maximal-ratio combining over nonidentical Weibull fading channels," IEEE Trans. Wireless Commun., vol. 4, no. 3, pp. 841-846, May 2005. 
[9] M. H. Ismail and M. M. Matalgah, "Performance of dual maximal ratio combining diversity in nonidentical correlated Weibull fading channels using padé approximation," Electron. Letters, vol. 32, no. 19, pp. 1752-1754, Sept. 1996.

[10] A. A. Abu-Dayya and N. C. Beaulieu, "Switched diversity on microcellular Ricean channels," IEEE Trans. Veh. Technol., vol. 43, no. 4, pp. 970-976, Nov. 1994.

[11] Y. C. Ko, M.-S. Alouini, and M. K. Simon, "Analysis and optimization of switched diversity systems," IEEE Trans. Veh. Technol., vol. 49, no. 5, pp. 1813-1831, Sept. 2000.

[12] C. Tellambura, A. Annamalai, and V. K. Bhargava, "Unified analysis of switched diversity systems in independent and correlated fading channels," IEEE Trans. Commun., vol. 49, no. 11, Nov. 2001.

[13] G. Femenias, "Reference-based dual switch and stay diversity systems over correlated Nakagami fading channels," IEEE Trans. Veh. Technol., vol. 52, no. 4, 2003.

[14] D. A. Zogas and G. K. Karagiannidis, "Infinite series representations associated with the bivariate Ricean distribution and their applications," IEEE Trans. Commun., vol. 53, no. 11, Nov. 2005.

[15] I. S. Gradshteyn and I. M. Ryzhik, Table of Integrals, Series, and Products, 6th ed. New York: Academic Press, 2000.

[16] A. A. Abu-Dayya and N. C. Beaulieu, "Analysis of switched diversity systems on generalized-fading channels," IEEE Trans. Commun., vol. 42, no. 11, pp. 2959-2964, Nov. 1994.

[17] A. Papoulis, Probability, Random Variables, and Stochastic processes, 2nd ed. McGraw-Hill, 1984.

[18] N. C. Sagias and G. K. Karagiannidis, "Gaussian class multivariate Weibull distributions: theory and applications in fading channels," IEEE Trans. Inform. Theory, vol. 51, no. 10, pp. 3608-3619, Oct. 2005. 